\title{
Synthesis and Characterization of PVP-Stabilized Palladium Nanoparticles by XRD, SAXS, SP-ICP-MS, and SEM
}

\author{
Katharina Walbrück $\mathbb{D}^{1},{ }^{1}$ Fabian Kuellmer, ${ }^{2}$ Steffen Witzleben $\mathbb{D}^{1},{ }^{1}$ and Klaus Guenther ${ }^{2,3}$ \\ ${ }^{1}$ Department of Natural Sciences, Bonn-Rhein-Sieg University of Applied Sciences, von-Liebig-Straße 20, \\ D-53359 Rheinbach, Germany \\ ${ }^{2}$ Institute of Nutritional and Food Sciences, Food Chemistry, Rheinische Friedrich-Wilhelms-University Bonn, Endenicher Allee 11- \\ 13, D-53115 Bonn, Germany \\ ${ }^{3}$ Institute of Bio- and Geosciences (IBG-2), Research Centre Jülich, Member of the Helmholtz Association of German \\ Research Centres, D-52425 Jülich, Germany
}

Correspondence should be addressed to Steffen Witzleben; steffen.witzleben@h-brs.de

Received 10 December 2018; Accepted 27 February 2019; Published 17 April 2019

Academic Editor: Vincenzo Baglio

Copyright (c) 2019 Katharina Walbrück et al. This is an open access article distributed under the Creative Commons Attribution License, which permits unrestricted use, distribution, and reproduction in any medium, provided the original work is properly cited.

\begin{abstract}
Due to increased emissions of palladium nanoparticles in recent years, it is important to develop analytical techniques to characterize these particles. The synthesis of defined and stable particles plays a key role in this process, as there are not many materials commercially available yet which could act as reference materials. Polyvinylpyrrolidone- (PVP-) stabilized palladium nanoparticles were synthesized through the reduction of palladium chloride by tetraethylene glycol (TEG) in the presence of $\mathrm{KOH}$. Four different methods were used for particle size analysis of the palladium nanoparticles. Palladium suspensions were analyzed by scanning electron microscopy (SEM), small angle X-ray scattering (SAXS), single-particle ICP-MS (SP-ICP-MS), and X-ray diffraction (XRD). Secondary particles between $30 \mathrm{~nm}$ and $130 \mathrm{~nm}$ were detected in great compliance with SAXS and SP-ICP-MS. SEM analysis showed that the small particulates tend to form agglomerates.
\end{abstract}

\section{Introduction}

Nanomaterials describe, in principle, materials with at least one dimension in the size range of $1 \mathrm{~nm}$ to $100 \mathrm{~nm}$. In particular, due to their unique properties (optical, chemical, mechanical, etc.), they find applications in numerous of industrial and commercial fields. Nanomaterials show great potential in fields of cosmetic, textiles, and medical and healthcare products as well as a food ingredient or in food packaging [1].

Nanomaterials are synthesized using two routes: topdown and bottom-up. In top-down approaches, nanomaterials are produced through restructuring a bulk material via mechanical milling, laser ablation, or electroexplosion. In contrast, bottom-up approaches synthesized nanomaterials through a chemical reaction out of small building blocks [2].
The stabilization of nanoparticles represents one key challenge in nanomaterial synthesis. Based on their thermodynamic instability, nanoparticles tend to form compact agglomerates. In consequence, the particles need to be stabilized. For nanoparticle stabilization, a variety of different methods are available. The most common method for stabilization is the use of a polymeric stabilizer such as polyvinylpyrrolidone (PVP). In his review, Cookson gives an overview about different types of stabilizers for nanoparticles [3].

Another key challenge represents the formation of controlled particle sizes and shapes during the synthesis. Therefore, the characterization of particle size and shape has been a very important research focus [4-6].

Metal-based nanoparticles have received considerable attention mainly due to their outstanding catalytic, electronic, magnetic, and optical properties [7]. Besides gold 
and silver, palladium nanoparticles are also of great importance. In particular, the small size makes them attractive for catalytic applications. Due to their large surface-to-volume ratio, they offer a higher catalytic potential than the bulk material [3].

In general, platinum group metals (PGM) such as platinum, rhodium, and palladium gained increasing interest in environmental research as they are emitted for example by exhaust fumes into the environment. They act as the catalytic active compounds in catalytic converters of cars. Because of its solubility, palladium can be mobilized into the environment and become biologically available and therefore can have an effect on flora and fauna [8]. It is commonly accepted that the exhausted particulate matter contains PGM nanoparticles that could pose a threat and have a not yet fully explored negative effect on health and environment [9]. Kińska et al. showed that Pd nanoparticles are taken up and accumulated in plant tissues such as Sinapis alba [10] but seem to not have an influence on plant growth and morphology.

Compared to other PGM, palladium is attractive because of its lower cost and its high activity towards oxygen reduction reactions [11]. In addition, the hydrogen storage properties of Pd make it an interesting material for many research areas [12], which might even lead to a higher emission rate of palladium in the future.

In the synthesis of defined and stable palladium nanoparticles, in particular, the application of different analytical characterization methods plays a key role. The synthesis of shape- and size-controlled palladium nanoparticles in combination with different analytical techniques has been studied only in a few examinations $[3,4,6]$. Yu et al. synthesized palladium icosahedra nanocrystals under microwave irradiation. The particle size determined with XRD, SEM, and TEM was about $31.7 \mathrm{~nm}$. Further methods for sample characterization were XPS (X-ray photoelectron spectroscopy) and UVvis spectroscopy [6].

Because of the increased use of palladium nanoparticles, it is important to establish more combination sets of analytical methods for the characterization of size and shape of these nanoparticles. Also, the synthesis of palladium nanoparticles will play a key role in that process because reference materials and analytical standards will be needed. Therefore, this paper is focused on the synthesis and particle size characterization of palladium nanoparticles. Palladium nanoparticles were synthesized under different conditions and investigated using four different methods.

\section{Materials and Methods}

2.1. Chemicals. Polyvinylpyrrolidone (PVP, $\mathrm{MW}=8000$ g/mol, Alfa Aesar)-stabilized palladium nanoparticles were synthesized through the reduction of palladium chloride $\left(\mathrm{PdCl}_{2}\right.$, Alfa Aesar) by tetra ethylene glycol (TEG, Merck) in the presence of potassium hydroxide ( $\mathrm{KOH}$, Merck). $\mathrm{PdCl}_{2}, \mathrm{PVP}$, and $\mathrm{KOH}$ were dissolved in TEG to produce the concentrations of the corresponding solutions. In addition, acetone $\left(\mathrm{C}_{3} \mathrm{H}_{6} \mathrm{O}, \mathrm{VWR}\right)$ was used to wash the synthesized palladium nanoparticles.
TABLE 1: Operational parameters for SP-ICP-MS analysis.

\begin{tabular}{lc}
\hline Software & $\begin{array}{c}\text { SyngistixTM, SyngistixTM Nano } \\
\text { Application Module } \\
\text { Element }\end{array}$ \\
Plow rate & $\rho=12.023 \mathrm{~g} / \mathrm{cm}^{3}$ \\
Transport efficiency & $0.306 \mathrm{~mL} / \mathrm{min}$ \\
Dwell time & $4.38 \%$ \\
Measurement time & $50 \mu \mathrm{s}$ \\
Mass fraction & $45 \mathrm{~s}$ \\
Ionization efficiency & $100 \%$ \\
\hline
\end{tabular}

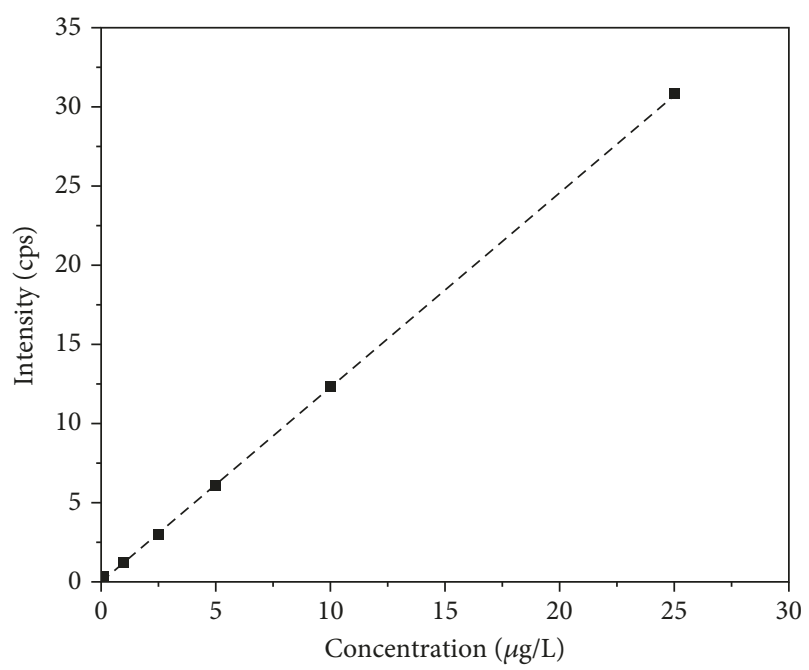

Figure 1: Calibration curve.

2.2. Synthesis of PVP-Pd NPs in an Autoclave under Microwave Irradiation. Palladium nanoparticles were synthesized by the chemical reduction of palladium chloride with TEG under microwave irradiation. Therefore, $5 \mathrm{~mL}$ of a $0.03 \mathrm{~mol} \mathrm{~L}^{-1} \mathrm{PdCl}_{2}$ solution, (a) $5 \mathrm{~mL}$ of a $0.15 \mathrm{~mol} \mathrm{~L}^{-1}$ or (b) $8 \mathrm{~mL}$ of a $0.10 \mathrm{~mol} \mathrm{~L}^{-1} \mathrm{PVP}$ solution (in monomeric unit), and $5 \mathrm{~mL}$ of (a) $0.05 \mathrm{~mol} \mathrm{~L}^{-1}$ or (b) $0.16 \mathrm{~mol} \mathrm{~L}^{-1} \mathrm{KOH}$ solution were added to a Teflon digestion vessel. Then, TEG was added to receive a final volume of $50 \mathrm{~mL}$. After stirring, the digestion vessel was placed in the microwave oven and heated for $60 \mathrm{~s}$ with $800 \mathrm{~W}$. The resultant solution was centrifuged $(3300 \mathrm{~g})$ and washed two times with acetone to separate the nanoparticles. Afterwards, the nanoparticles were redispersed in acetone.

2.3. Synthesis of PVP-Pd NPs under Normal Pressure. In a $100 \mathrm{~mL}$ round bottom flask, $32 \mathrm{~mL}$ TEG was heated at $130^{\circ} \mathrm{C}$. After $5 \mathrm{~h}$, (c) $5 \mathrm{~mL}$ or (d) $10 \mathrm{~mL}$ of a $0.03 \mathrm{~mol} \mathrm{~L}^{-1} \mathrm{PdCl} 2$ solution and (c) $8 \mathrm{~mL}$ or (d) $5 \mathrm{~mL}$ of a $0.10 \mathrm{~mol} \mathrm{~L}^{-1} \mathrm{PVP}$ solution (in monomeric unit) and $5 \mathrm{~mL}$ of a $0.16 \mathrm{~mol} \mathrm{~L}^{-1}$ $\mathrm{KOH}$ solution were added dropwise, keeping temperature and stirred for $20 \mathrm{~h}$. Afterwards, the resulting solution was centrifuged (3300g) and washed two times with acetone to separate the nanoparticles. Finally, the nanoparticles were redispersed in acetone. 
TABLE 2: Operational parameters for XRD analysis.

\begin{tabular}{lc}
\hline Software & $\begin{array}{c}\text { Bruker Diffrac.XRD (Version 4.0) } \\
\text { Bruker Diffrac.EVA (Version 4.1) }\end{array}$ \\
Scan type & Coupled two theta/theta \\
Theta & $20^{\circ}-42.5^{\circ}$ \\
2 theta & $40^{\circ}-85^{\circ}$ \\
Step size & $15^{\circ}$ \\
Time per step & $240 \mathrm{~s}$ \\
Measurement time & $960 \mathrm{~s}$ \\
Steps & 4 \\
Collimator & $0.5 \mathrm{~mm}$ \\
\hline
\end{tabular}

TABLE 3: Operational parameters for SAXS analysis.

\begin{tabular}{lc}
\hline Software & $\begin{array}{c}\text { Bruker Diffrac.XRD (Version 4.1) } \\
\text { Bruker Diffrac.EVA (Version 4.0) } \\
\text { Bruker NanoFit (Version 1.2.1) }\end{array}$ \\
Scan type & Still (VÅTEC-500) \\
Theta & $0^{\circ}$ \\
2 theta & $0^{\circ}$ \\
Measurement time & $90 \mathrm{~s}$ \\
Collimator & $0.5 \mathrm{~mm}$ \\
\hline
\end{tabular}

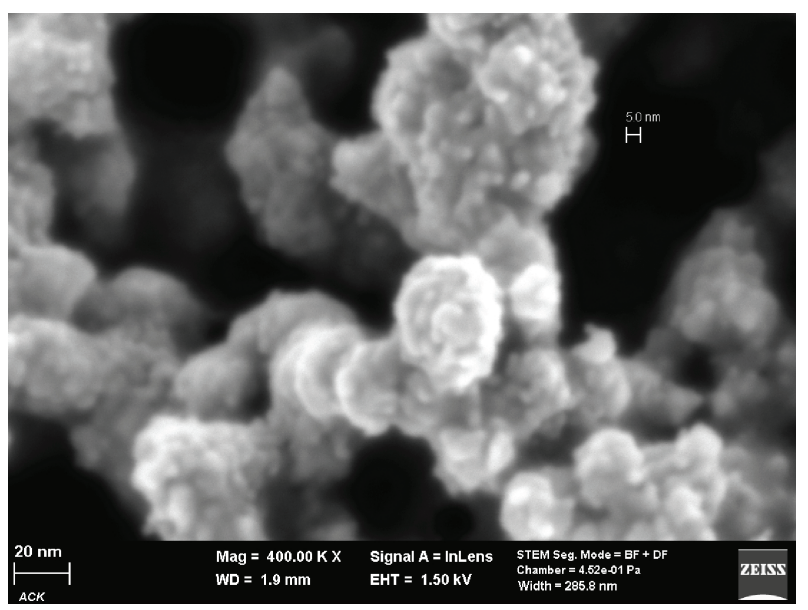

Figure 2: SEM image of approach (a).

2.4. Characterization. Four different methods were used for particle size analysis of the synthesized palladium nanoparticles. The nanoparticles were analyzed by scanning electron microscopy (SEM), small angle X-ray scattering (SAXS), single-particle ICP-MS (SP-ICP-MS), and X-ray diffraction (XRD).

2.4.1. Scanning Electron Microscopy (SEM). The SEM observations were performed on Zeiss GeminiSEM 500. The nanoparticles were dispersed in acetone, and a drop of this suspension was deposited onto a carbon specimen sample holder.

2.4.2. Single-Particle ICP-MS. NexIon 350D ICP-MS (PerkinElmer) in single-particle mode was used for the analysis of

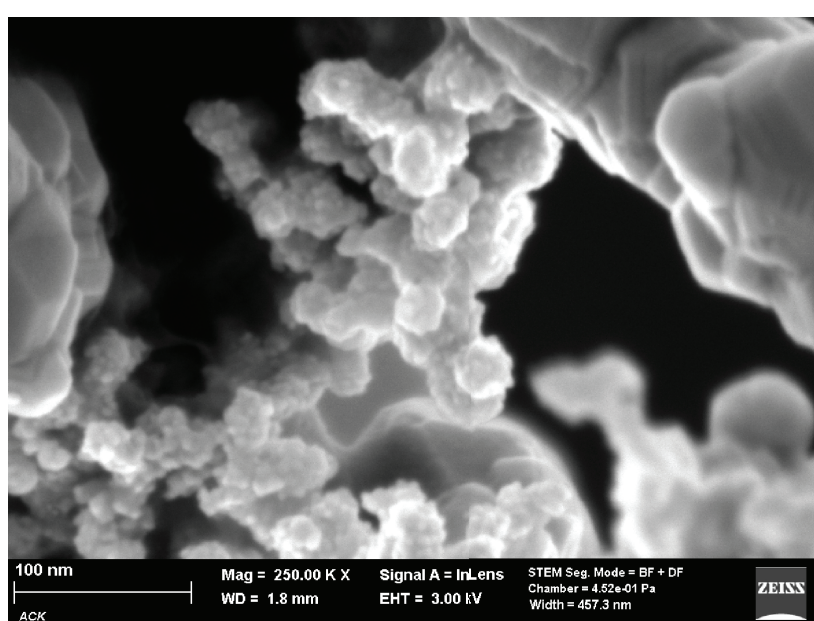

Figure 3: SEM image of approach (d).

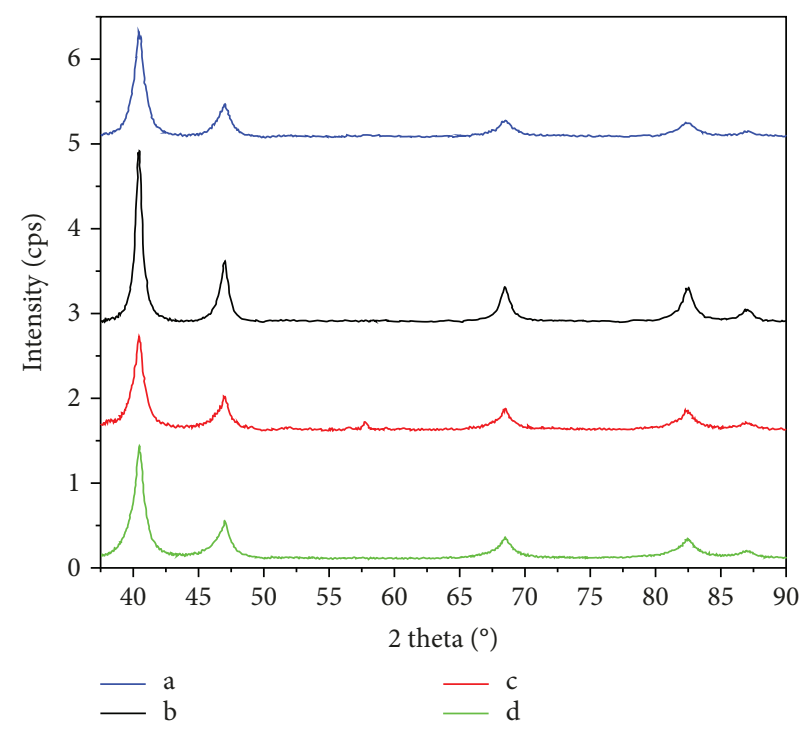

FIgURE 4: XRD pattern of approaches (a)-(d).

TABle 4: Crystallographic data.

\begin{tabular}{|c|c|c|c|c|c|}
\hline Structure parameters & \multicolumn{5}{|c|}{ Palladium nanoparticles } \\
\hline Lattice system & \multicolumn{5}{|c|}{ Cubic, face-centered } \\
\hline Space group & \multicolumn{5}{|c|}{ Fm-3m } \\
\hline Volume of the primitive cell & \multicolumn{5}{|c|}{$57.8 \AA$} \\
\hline Primitive cell & \multicolumn{5}{|c|}{$\begin{array}{c}\alpha=\beta=\gamma=90^{\circ} \\
\mathrm{a}=\mathrm{b}=\mathrm{c}=3.867 \pm 0.003 \AA\end{array}$} \\
\hline Scattering angles & $40.5^{\circ}$ & $47.0^{\circ}$ & $68.5^{\circ}$ & $82.4^{\circ}$ & $87.0^{\circ}$ \\
\hline Miller indices (hkl) & (111) & $(200)$ & $(220)$ & $(311)$ & $(222)$ \\
\hline
\end{tabular}

palladium nanoparticles. The instrumental parameters are summarized in Table 1. A multielement standard containing $1 \mu \mathrm{g} / \mathrm{L} \mathrm{Be}, \mathrm{Ce}, \mathrm{Fe}, \mathrm{In}, \mathrm{Li}, \mathrm{Mg}, \mathrm{Pd}$, and $\mathrm{U}$ in $1 \%$ nitric acid was used for optimal sensitivity and precision. A $1000 \mathrm{ppb}$ stock solution of ${ }^{106} \mathrm{Pd}$ was prepared by dissolution of $50 \mu \mathrm{L}$ palladium standard and $6.75 \mathrm{~mL}$ hydrochloric acid in ultrapure water. Working solutions of $0.1,1,2.5,5$, and $25 \mathrm{ppb}$ were 
TABLE 5: XRD results.

\begin{tabular}{lccc}
\hline Sample & 2 theta $\left(^{\circ}\right)$ & FWHM & Crystallite size $(\mathrm{nm})$ \\
\hline (a) & $87.1^{\circ} \pm 0.1^{\circ}$ & $1.1 \pm 0.1$ & $12.4 \pm 0.3$ \\
(b) & $87.0^{\circ} \pm 0.1^{\circ}$ & $1.1 \pm 0.1$ & $13.6 \pm 0.8$ \\
(c) & $87.0^{\circ} \pm 0.1^{\circ}$ & $1.1 \pm 0.1$ & $11.9 \pm 0.6$ \\
(d) & $86.9^{\circ} \pm 0.1^{\circ}$ & $1.1 \pm 0.1$ & $12.1 \pm 0.7$ \\
\hline
\end{tabular}

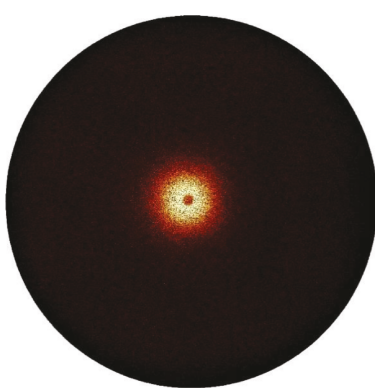

(a)

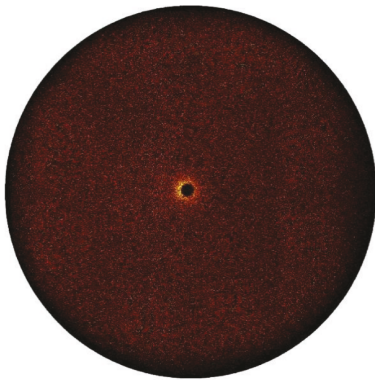

(c)

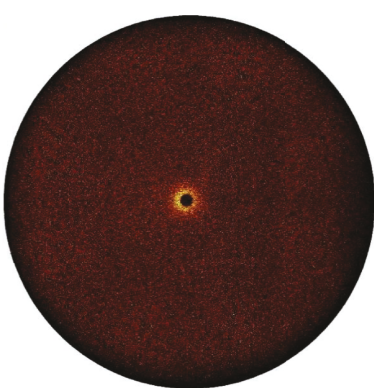

(b)

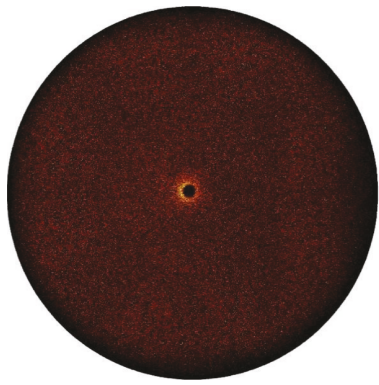

(d)
Figure 5: SAXS pattern.

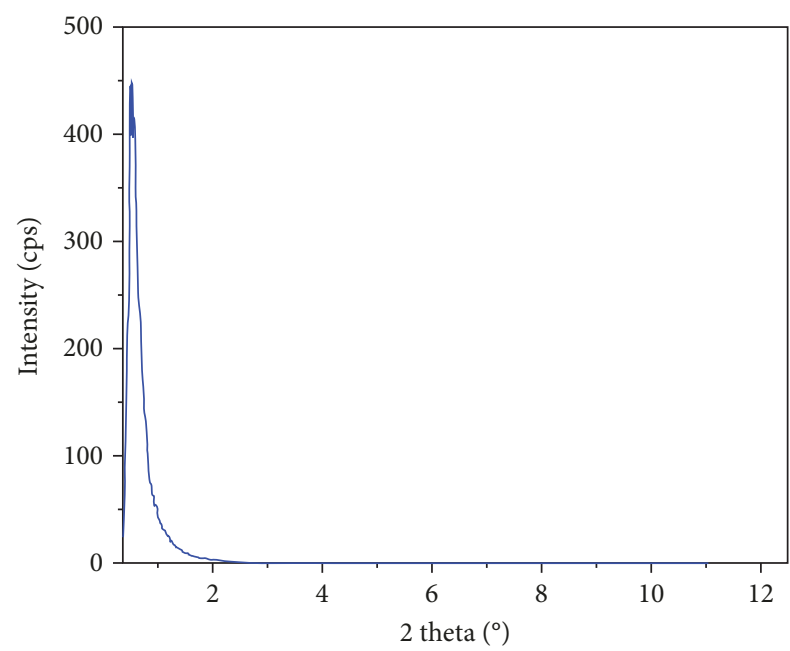

$-\mathrm{a}$

FIGURE 6: SAXS curve of approach (a).

prepared for calibration. The achieved calibration curve (Figure 1) was $y=1.2329 x+0.0206, R^{2}=0.9999$. The samples were diluted in ultrapure water until the particle concentration is in the range of about $50,000 \mathrm{P} / \mathrm{mL}$. The transport efficiency was determined with a gold particle suspension

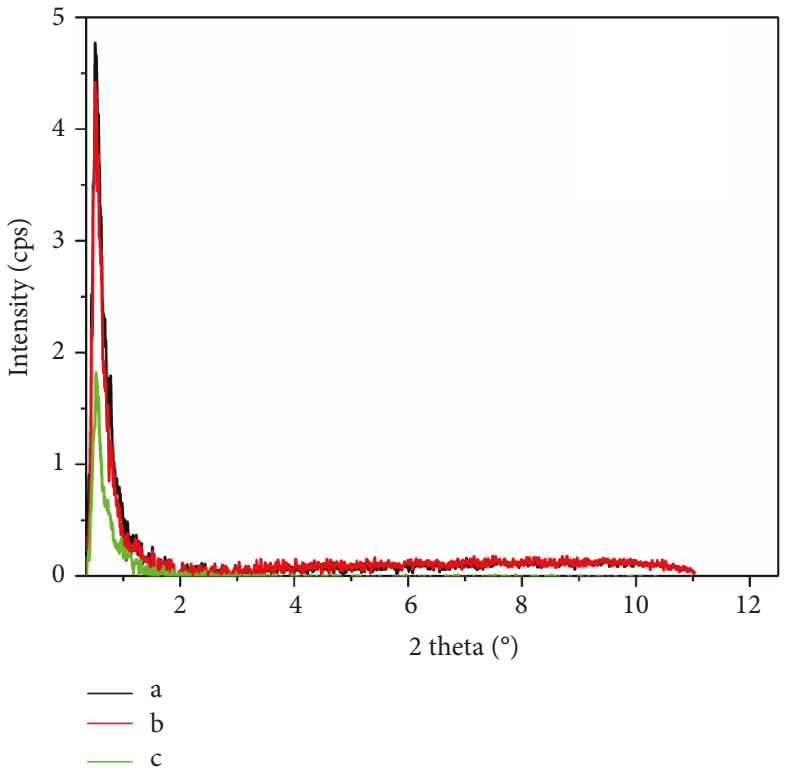

FIGURE 7: SAXS curve of approaches (b)-(d).

TABLE 6: SAXS results.

\begin{tabular}{lcccc}
\hline Sample & $\begin{array}{c}\text { Intensity } \\
(\mathrm{cps})\end{array}$ & 2 theta $\left(^{\circ}\right)$ & $d(\AA)$ & $\begin{array}{c}\text { Particle diameter } \\
(\mathrm{nm})\end{array}$ \\
\hline (a) & $435.0 \pm 4.4$ & $0.50 \pm 0.01$ & $176.1 \pm 5.2$ & $113.0 \pm 0.1$ \\
(b) & $4.8 \pm 0.6$ & $0.49 \pm 0.01$ & $177.8 \pm 5.7$ & $131.3 \pm 0.8$ \\
(c) & $4.0 \pm 2.6$ & $0.50 \pm 0.01$ & $176.1 \pm 5.2$ & $45.0 \pm 1.7$ \\
(d) & $1.6 \pm 0.1$ & $0.51 \pm 0.01$ & $173.1 \pm 2.9$ & $34.5 \pm 0.5$ \\
\hline
\end{tabular}

TABLE 7: SP-ICP-MS results.

\begin{tabular}{lc}
\hline Sample & Particle diameter $(\mathrm{nm})$ \\
\hline (a) & 113.0 \\
(b) & 132.0 \\
(c) & 42.2 \\
(d) & 18.5 \\
\hline
\end{tabular}

using the particle frequency method as described by Pace et al. [13].

2.4.3. X-Ray Diffraction (XRD). X-ray diffraction analysis was carried out with a Bruker D8 Discover diffractometer, with a $\mathrm{Cu}$ microfocus $\mathrm{X}$-ray source $(\lambda=0.15418 \mathrm{~nm})$ and a 2dimensional Vantec 500 detector. A drop of the nanoparticle suspension was deposited onto a glass slide. The measurements were performed in a 2-theta range from $37^{\circ}$ to $90^{\circ}$, with an exposure time of $960 \mathrm{~s}$. Further instrumental parameters are given in Table 2 . The data analysis was performed using the program Diffrac.Eva (Bruker).

2.4.4. Small Angle X-Ray Scattering (SAXS). SAXS measurements were also performed on a Bruker D8 Discover diffractometer, with a $\mathrm{Cu}$ microfocus $\mathrm{X}$-ray source and a 2-dimensional Vantec 500 detector. Further instrumental parameters are given in Table 3 . The synthesized $\mathrm{Pd}$ 


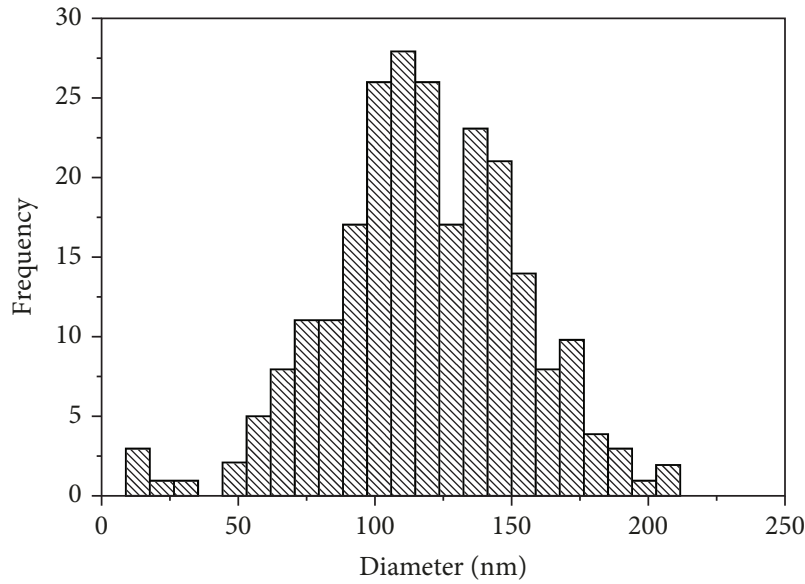

(a)

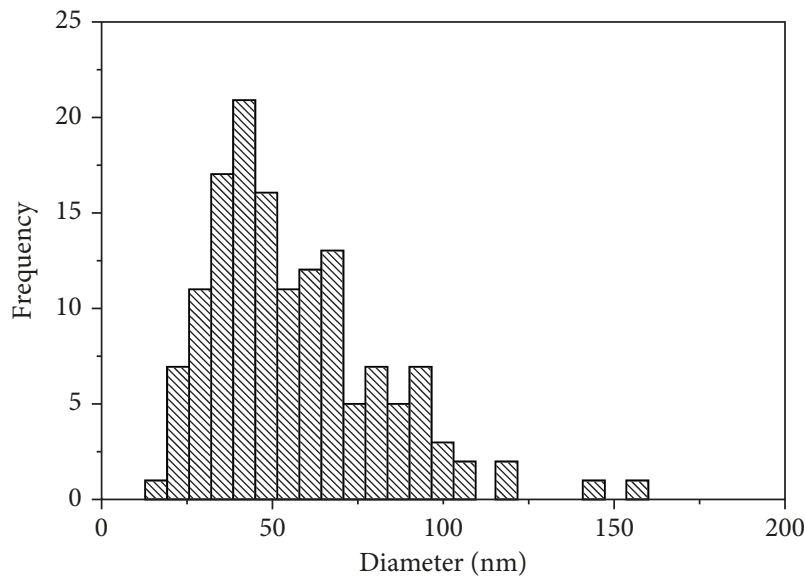

(c)

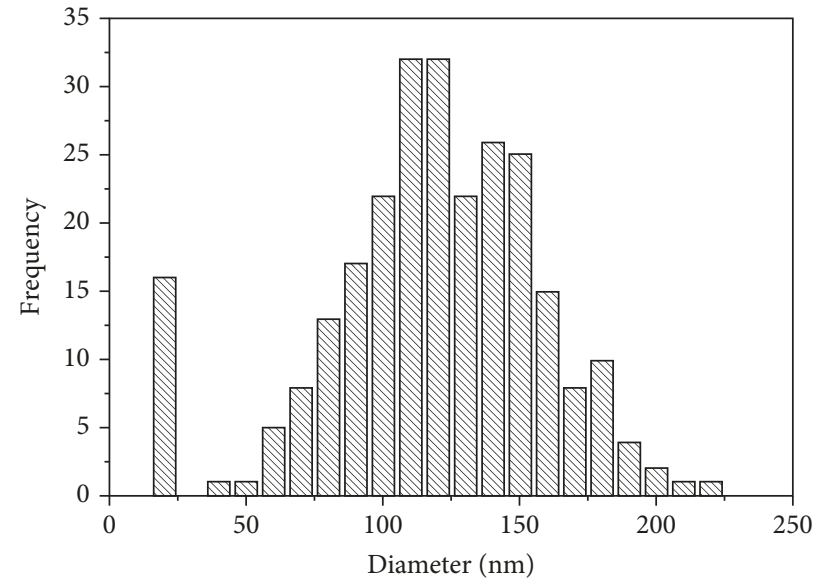

(b)

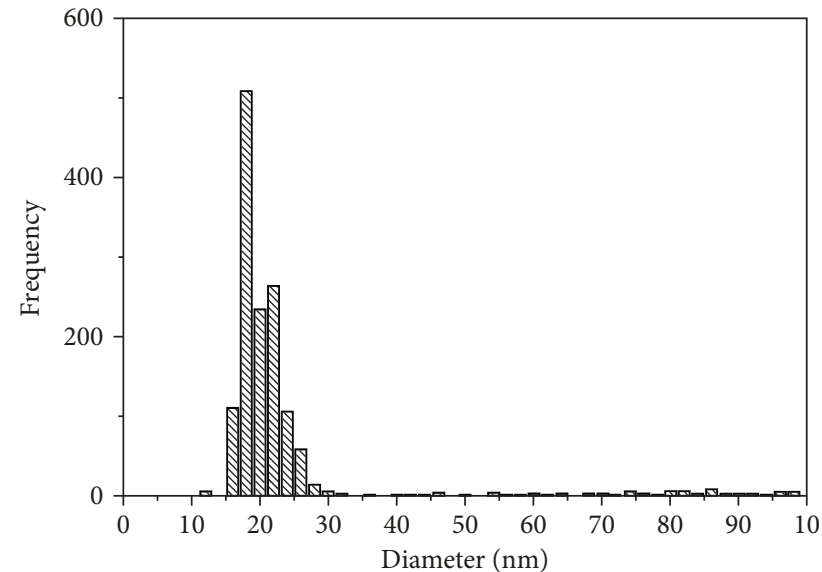

(d)

FIgURE 8: Histograms of approaches (a)-(d).

nanoparticle suspensions were injected into a quartz glass capillary and mounted on a capillary sample holder. The measurements were performed in transmission mode, with an exposure time of $90 \mathrm{~s}$. The SAXS data was analyzed using the program Diffrac.Eva (Bruker) and NanoFit (Bruker).

\section{Results and Discussion}

3.1. SEM. Figures 2 and 3 present the SEM images of palladium nanoparticles, which show that most of the particles have a spherical shape with an average particle size in the range $5-15 \mathrm{~nm}$. In addition, the SEM images indicate the formation of agglomerates.

3.2. $X R D$. The $\mathrm{X}$-ray diffractogram of the synthesized palladium nanoparticles is shown in Figure 4. As can be seen, the diffraction pattern of the palladium nanoparticles is showing diffraction peaks positioned at the Bragg angles $(2 \Theta) 40.5^{\circ}, 47.0^{\circ}, 68.5^{\circ}, 82.4^{\circ}$, and $87.0^{\circ}$. The observed peaks correspond to the lattice planes (111), (200), (311), and (222). All diffraction peaks can be well indexed to a facecentered cubic lattice system according to the COD database (COD ID: 1011112). The structure parameters of the synthesized palladium nanoparticles are listed in Table 4.
The crystallite size, $L$, was calculated using the Scherrer equation

$$
L=\frac{K \lambda}{\text { FWHM } \cos \theta},
$$

where $K=0.9$ is the Scherrer factor, $\lambda$ the wavelength, FWHM is the full width at half maximum of the peak, and $\theta$ the Bragg angle. Table 5 summarizes the obtained crystallite sizes.

3.3. SAXS. Small angle X-ray scattering is also a powerful method for the determination of size and shape of nanoparticles. Figure 5 shows the SAXS patterns of the synthesized palladium nanoparticles. Around the beam stop in the center an isotropic scattering was observed. Compared to the SAXS patterns (b)-(d), an intensive scattering corona around the beam stop could be observed for sample (a).

The integration of the SAXS pattern led to the SAXS curves (Figures 6 and 7). The intensity of sample (a) is about 100 times higher than for samples (b)-(d).

These data were used for the calculation of the particle diameter $[14,15]$ with the program NanoFit (Bruker). The fit of SAXS curves revealed nanoparticle diameters between 
TABLE 8: Summary of all results.

\begin{tabular}{lcccc}
\hline Sample & SEM $(\mathrm{nm})$ & XRD $(\mathrm{nm})$ & SAXS $(\mathrm{nm})$ & SP-ICP-MS $(\mathrm{nm})$ \\
\hline (a) & $5-10$ & $12.1 \pm 0.7$ & $113.0 \pm 0.1$ & 113.0 \\
(b) & $/$ & $12.4 \pm 0.3$ & $131.3 \pm 0.8$ & 132.0 \\
(c) & $/$ & $13.6 \pm 0.8$ & $45.0 \pm 1.7$ & 42.2 \\
(d) & 15 & $11.9 \pm 0.6$ & $34.5 \pm 0.5$ & 18.5 \\
\hline
\end{tabular}

$34 \mathrm{~nm}$ and $132 \mathrm{~nm}$. The particle size of sample (a) is determined as $113.0 \pm 0.1 \mathrm{~nm}$. In contrast, sample (d) revealed a particle size of $34.5 \pm 0.5 \mathrm{~nm}$. In Table 6 , the resulting particle sizes of all SAXS measurements are summarized.

3.4. SP-ICP-MS. The analysis of the synthesized palladium nanoparticles performed by SP-ICP-MS is summarized in Table 7. Figure 8 presents the obtained histograms for the analyzed palladium nanoparticles. Nanoparticles in the range $30 \mathrm{~nm}$ to $132 \mathrm{~nm}$, with broad size distributions, were obtained by the different synthesis. Even though the method was not validated because of the lack of an appropriate reference material, the results were clearly in compliance with the SAXS results.

\section{Conclusion}

The synthesis of defined and stable particles plays a key role in the characterization process of nanoparticles. Currently, no reference materials for palladium nanoparticle are commercially availability. Therefore, two preparation methods and different concentrations of PVP-stabilized palladium nanoparticles were evaluated. In order to determine the particle size of the synthesized particles, four different measuring methods have been used. However, the results obtained by the different measuring methods cannot be rigorously compared because these techniques do not measure the same size parameter. SEM allows the determination of the primary particle size, whereas SP-ICP-MS and SAXS measure the mean particle diameter, and the diameters calculated from XRD measurements indicate the crystallite size.

The synthesis of Pd nanoparticles with the chosen preparation techniques was successful. Table 8 presents a comparative overview of all results. Nanoparticles tend to form relatively compact agglomerates. In particular, the particle size determined by SAXS and SP-ICP-MS was highly affected by the accumulation of agglomerates. In consequence, mostly secondary particles were detected by the use of these methods. To prevent that from happening, the samples might need to be sonicated prior to the measurement. The results obtained from both methods are in good compliance with each other. The results of the SAXS and SP-ICP-MS measurements allow also to distinguish between the two preparation methods. The samples (a) and (b) synthesized in an autoclave under microwave irradiation have a higher particle size than the samples prepared under normal pressure.

The SEM images indicate the formation of agglomerated small particles with a primary particle size in the range of $5 \mathrm{~nm}$ to $15 \mathrm{~nm}$, which are in good agreement with the XRD results.

The nanoparticles synthesized in this work are suitable to serve as the first step in the production of reference materials for the improvement of analytical methods. Further studies are necessary to investigate the stabilization and stability of the Pd NPs.

\section{Data Availability}

The data used to support the findings of this study are available from the corresponding author upon request.

\section{Conflicts of Interest}

The authors declare that there is no conflict of interest regarding the publication of this paper.

\section{Acknowledgments}

The measurement equipment Bruker D2 and Bruker D8 was funded by the Federal Ministry of Education and Research Germany.

\section{References}

[1] G. Lövestam, H. Rauscher, G. Roebben et al., "Considerations on a definition of nanomaterial for regulatory purposes," Joint Research Centre (JRC) Reference Reports, 2010.

[2] C. Laura, B. M. Luisa, M. J. Ángel, G. F. González, and B. Antonio, "Mechanism and applications of metal nanoparticles prepared by bio-mediated process," Reviews in Advanced Sciences and Engineering, vol. 3, no. 3, pp. 199-216, 2014.

[3] J. Cookson, "The preparation of palladium nanoparticles," Platinum Metals Review, vol. 56, no. 2, pp. 83-98, 2012.

[4] N. V. Long, T. Hayakawa, T. Matsubara, N. D. Chien, M. Ohtaki, and M. Nogami, "Controlled synthesis and properties of palladium nanoparticles," Journal of Experimental Nanoscience, vol. 7, no. 4, pp. 426-439, 2012.

[5] G. Steinborn, M. Gemeinert, and W. Schmidt, "Vergleich verschiedener messverfahren zur partikelgrößenanalyse am beispiel von nanodispersem $\mathrm{ZrO} 2$-pulver," Chemie Ingenieur Technik, vol. 88, no. 7, pp. 984-994, 2016.

[6] Y. Yu, Y.Zhao, T. Huang, and H. Liu, "Shape-controlled synthesis of palladium nanocrystals by microwave irradiation," Pure and Applied Chemistry, vol. 81, no. 12, pp. 2377-2385, 2009.

[7] V. Leso and I. Iavicoli, "Palladium nanoparticles: toxicological effects and potential implications for occupational risk assessment," International Journal of Molecular Sciences, vol. 19, no. 2, p. 503, 2018.

[8] S. Zimmermann and B. Sures, "Significance of platinum group metals emitted from automobile exhaust gas converters for the biosphere," Environmental Science and Pollution Research, vol. 11, no. 3, pp. 194-199, 2004.

[9] S. Uibel, M. Takemura, D. Mueller, D. Quarcoo, D. Klingelhoefer, and D. A. Groneberg, "Nanoparticles and cars - analysis of potential sources," Journal of Occupational Medicine and Toxicology, vol. 7, no. 1, pp. 13-17, 2012.

[10] K. Kińska, J. Jiménez-Lamana, J. Kowalska, B. KrasnodębskaOstręga, and J. Szpunar, "Study of the uptake and bioaccumulation of palladium nanoparticles by Sinapis alba using single 
particle ICP-MS," Science of the Total Environment, vol. 615, pp. 1078-1085, 2018.

[11] S. Mukherjee, M. Carmo, G. Kumar, R. C. Sekol, A. D. Taylor, and J. Schroers, "Palladium nanostructures from multicomponent metallic glass," Electrochimica Acta, vol. 74, pp. 145-150, 2012.

[12] B. D. Adams and A. Chen, "The role of palladium in a hydrogen economy," Materials Today, vol. 14, no. 6, pp. 282-289, 2011.

[13] H. E. Pace, N. J. Rogers, C. Jarolimek, V. A. Coleman, C. P. Higgins, and J. F. Ranville, "Determining transport efficiency for the purpose of counting and sizing nanoparticles via single particle inductively coupled plasma mass spectrometry," Analytical Chemistry, vol. 83, no. 24, pp. 9361-9369, 2011.

[14] H. Benoit, "On the effect of branching and polydispersity on the angular distribution of the light scattered by Gaussian coils," Journal of Polymer Science, vol. 11, no. 5, pp. 507-510, 1953.

[15] J. S. Pedersen, "Form factors of block copolymer micelles with spherical, ellipsoidal and cylindrical cores," Journal of Applied Crystallography, vol. 33, no. 3, pp. 637-640, 2000. 


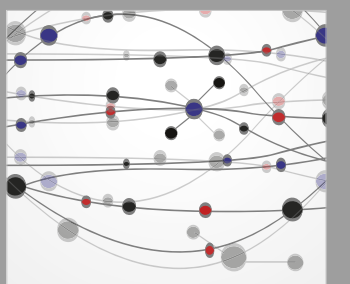

The Scientific World Journal
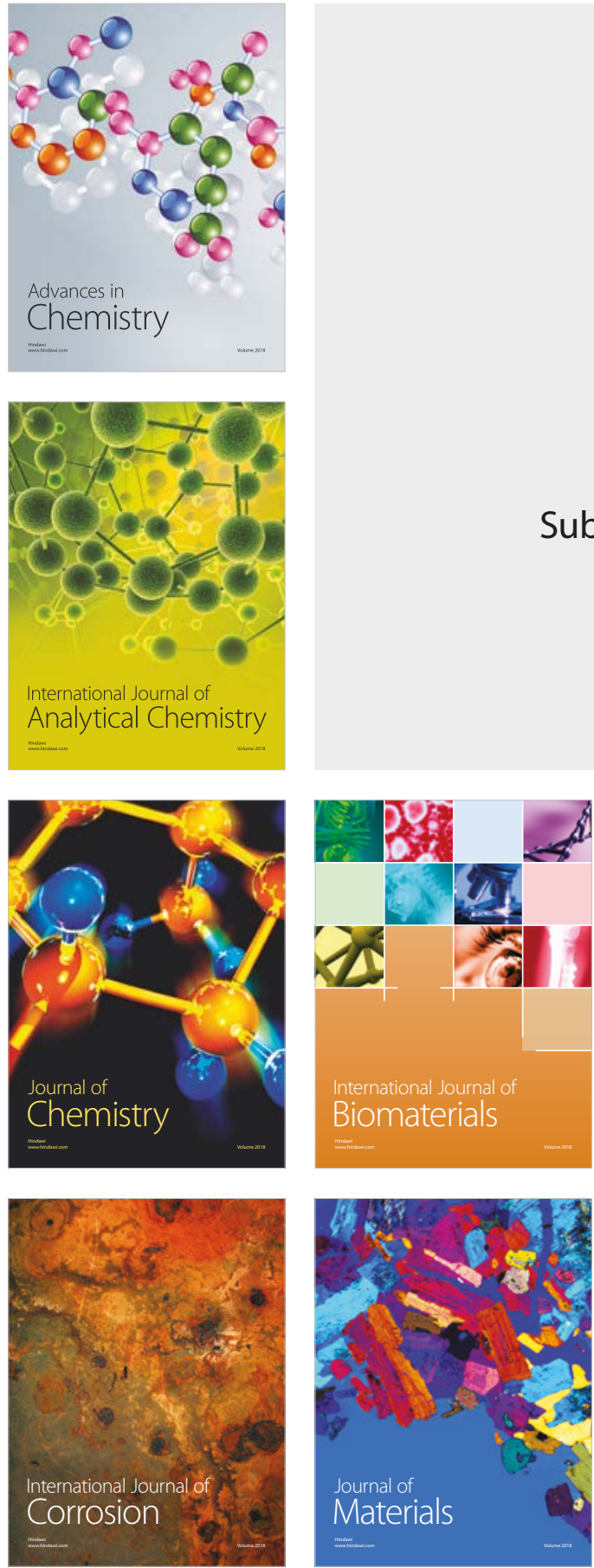

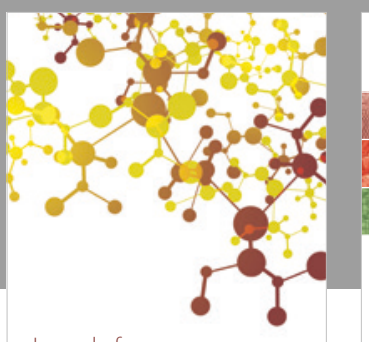

Journal of

Applied Chemistry
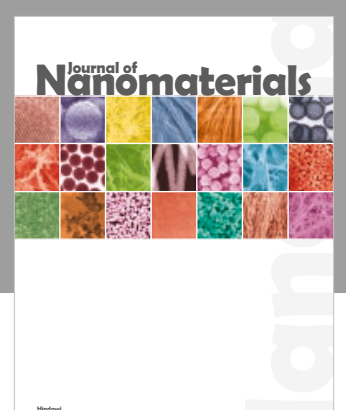

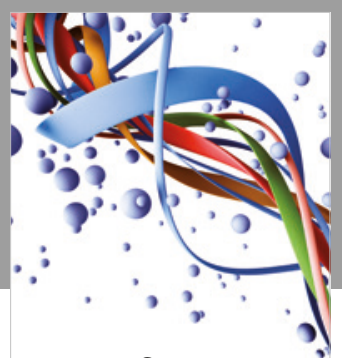

Scientifica

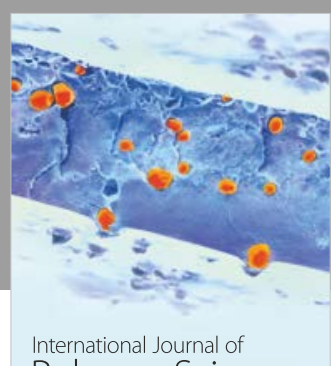

Polymer Science

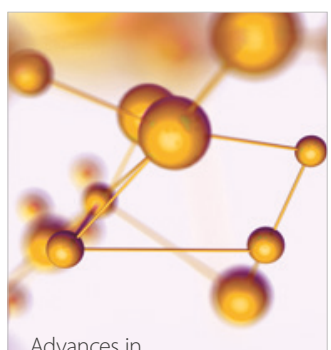

Physical Chemistry
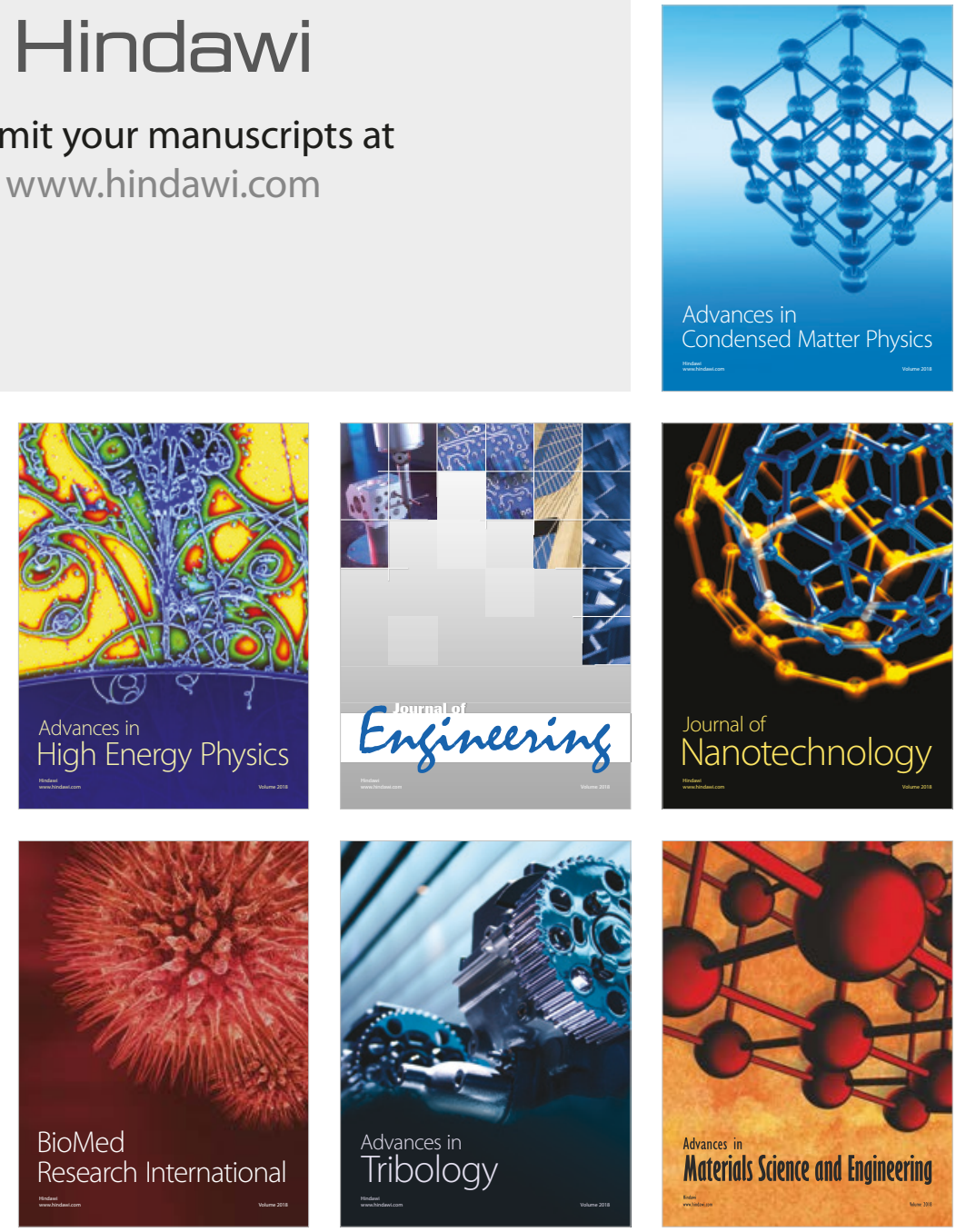